\title{
Ability of ambulatory ECG-based T-wave alternans to modify risk assessment of cardiac events: a systematic review
}

\author{
Xiao-Qing Quan ${ }^{1 \dagger}$, Hong-Lian Zhou' ${ }^{1 \dagger}$, Lei Ruan', Jia-Gao Lv², Ji-Hua Yao ${ }^{3}$, Feng Yao ${ }^{3}$, Kui Huang ${ }^{1}$ \\ and Cun-Tai Zhang ${ }^{1 *}$
}

\begin{abstract}
Background: Exercise-based spectral T-wave alternans (TWA) has been proposed as a noninvasive tool-identifying patients at risk of sudden cardiac death (SCD) and cardiac mortality. Prior studies have indicated that ambulatory electrocardiogram (AECG)-based TWA is an important alternative platform to exercise for risk stratification of cardiac events. This study sought to review data regarding 24-hour AECG-based TWA and to discuss its potential role in risk stratification of fatal cardiac events across a series of patient risk profiles.
\end{abstract}

Methods: Prospective clinical studies of the predictive value of AECG-based TWA obtained with daily activity published between January 1990 and November 2014 were retrieved. Major endpoints included composite endpoint of SCD, cardiac mortality, and severe arrhythmic events.

Results: Data were accumulated from 5 studies involving a total of 1,588 patients, including 317 positive and 1,271 negative TWA results. Compared with the negative group, positive group showed increased rates of SCD (hazard ratio [HR]: 7.49, 95\% confidence interval [Cl]: 2.65 to 21.15), cardiac mortality (HR: 4.75, 95\% Cl: 0.42 to 53.55), and composite endpoint (SCD, cardiac mortality, and severe arrhythmic events, HR: 5.94, 95\% Cl: 1.80 to 19.63). For the 4 studies evaluating TWA measured using the modified moving average method, the HR associated with a positive versus negative TWA result was 9.51 (95\% Cl: 4.99 to 18.11) for the composite endpoint.

Conclusions: The positive group of AECG-based TWA has a nearly six-fold risk of severe outcomes compared with the negative group. Therefore, AECG-based TWA provides an accurate means of predicting fatal cardiac events.

Keywords: T-wave alternans, Ambulatory electrocardiogram, Sudden cardiac death, Cardiac event

\section{Background}

The T-wave alternans (TWA) phenomenon, a repeating $A B A B A B$ pattern in the morphology and amplitude of the ST-segment or T-wave, has long been recognized and linked to arrhythmogenesis [1-3]. TWA has been proposed as noninvasive means of stratifying patients for the risk of sudden cardiac death (SCD) and cardiac mortality $[1,4,5]$.

TWA value can be obtained during exercise testing (bicycle or treadmill) or AECG recording [1,2,5]. Exercise-

\footnotetext{
*Correspondence: ctzhang@tjh.tjmu.edu.cn

${ }^{\dagger}$ Equal contributors

'Department of Geriatrics, Tongji Hospital, Tongji Medical College, Huazhong University of Science and Technology, Wuhan 430030, China

Full list of author information is available at the end of the article
}

based TWA has been mainly analyzed by frequencydomain spectral method $[1,2,4,6]$. A target heart rate range of 105 to 110 beats/min for a sustained period $(>2$ min) was determined for microvolt TWA assessed during exercise testing $[1,6]$. Therefore, not all patients can undergo a spectral TWA test, for example, patients taking medications such as beta-blockers, digoxin, and those with physical limitations. Recent study by Jackson et al. showed that spectral method was not widely applicable in patients hospitalized with acute decompensated heart failure, $49 \%$ were ineligible for spectral TWA testing, due either to physical inability to undertake the test, $\mathrm{AF}$, or pacemaker dependency [7]. In another study by Tapanainen et al., of the 379 consecutive post-acute myocardial infarction

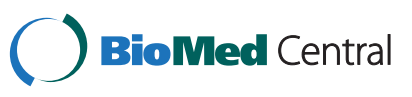

(c) 2014 Quan et al.; licensee BioMed Central. This is an Open Access article distributed under the terms of the Creative Commons Attribution License (http://creativecommons.org/licenses/by/4.0), which permits unrestricted use, distribution, and reproduction in any medium, provided the original work is properly credited. The Creative Commons Public Domain Dedication waiver (http://creativecommons.org/publicdomain/zero/1.0/) applies to the data made available in this article, unless otherwise stated. 
(AMI) patient population, TWA could be analyzed reliably during exercise test in only $53 \%$ of the cases [8].

Because of inability to achieve the target heart rate, the presence of excessive ectopy, nonsustained TWA, or excessive signal noise due to motion or respiration, there is a relatively high incidence ( $19 \%$ to $46 \%$ of all cases) of indeterminate test results $[1,7,9-11]$. For the prognostic value of indeterminate TWA results, the results was controversial. Some studies have suggested that an indeterminate TWA tests due to patient factors was a strong predictor of SCD or cardiac arrest $[8,10,12]$, but conflicting opinions exists $[7,9,13,14]$.

Ambulatory electrocardiogram (AECG) recording-based TWA represents an important alternative platform to stratify patients at risk for serious cardiac events $[1,2]$. TWA testing based on 24-hour Holter ECG recorded during daily activity does not require heart rate elevation and so overcomes some of the limitations of spectral method [5,15-17]. Prior studies with AECG-based TWA analysis have shown that an abnormal TWA result is modestly associated with cardiac events and that a normal result has a high negative predictive value [18-21]. However, negative studies have also appeared, including two prospective studies in patients with chronic heart failure [22] and AMI [23].

We therefore performed this systematic review and meta-analysis to determine the utility of 24-hour Holter AECG-based TWA for risk stratification of cardiac events in a wide variety of patient populations.

\section{Methods}

\section{Ethics statement}

The Ethics Committee (IRB) of Tongji Hospital (Wuhan City, Hubei Province, China) recognizes that this systematic review and meta-analysis of de-identified, publicly available data does not constitute "human subjects research" as defined by relevant national regulations, and therefore does not require Ethics Committee review.

\section{Search criteria}

A systematic review of the available literature was performed according to the PRISMA (preferred reporting items for systematic reviews and meta-analyses) guidelines (Additional file 1). This article evaluates the potential value of 24-hour Holter AECG recording-based TWA in risk stratification of fatal cardiac events in a wide variety of patient populations. We performed a systematic literature search for prospective studies published in English between January 1990 and November 2014. The search was restricted to full-text English publications and human subjects. Using the terms "T wave alternans", "ambulatory electrocardiogram (ECG)", "Holter", we searched the following databases: MEDLINE, EMBASE, Cochrane Library, International Network of Agencies for Health Technology
Assessment, and the Web of Science. A second search of articles published by authors identified in the initial search and a review of the bibliographies of all articles were performed to identify additional articles for review. We also hand-searched reviews and previous meta-analysis for additional potentially relevant studies.

We excluded review articles, abstracts, book chapters, conference proceedings, and correspondence. If more than one study reported on the same cohort, then we only used the most recent publication of that cohort. Articles of subcohorts were excluded if an article using the entire cohort was previously included. When the same research study was found in multiple journals or repositories, only one instance of the study was included.

\section{Inclusion criteria}

Studies were included if they met the following criteria: 1) prospective clinical study of $\geq 100$ human subjects; 2 ) 24-hour Holter AECG-based TWA obtained with daily activity; 3) reported meaningful clinical endpoints including SCD, cardiac mortality, and/or ventricular arrhythmias; 4) provided clear definition of positive or negative TWA. Duplicate publications and studies with a follow-up of less than 6 months were excluded.

Two investigators (Q.X. and Z.H.) independently reviewed the titles and abstracts of these articles and excluded those that clearly did not meet the inclusion criteria. A consensus was reached on which articles should be completely reviewed for potential inclusion in this study.

\section{Data extraction}

Two investigators (Q.X. and Z.H.) independently reviewed potential articles blinded to the author, journal, and institution. Data for each article were abstracted, including study design, inclusion and exclusion criteria, clinical characteristics of participants, details regarding TWA result, end points of the study, duration of follow-up, raw data when provided, and reported findings, including hazard ratios (HR).

\section{Quality analysis of study}

We assessed study quality based on the following assessment of the articles [9]: 1) the follow-up completed was beyond $90 \%$ of the cohort, 2) adjudication of outcomes was blinded to the results of TWA testing, and 3) a multivariate analysis using other standard predictors of cardiac events was performed. A study was considered at least of fair quality if it fulfilled the first criteria. A study was considered good quality if one of the other two criteria was met. This quality assessment was not used to assess study inclusion or exclusion, but was included as a study characteristic. 


\section{Statistical analysis}

The outcomes of each study were presented as HR with confidence interval (CI) of TWA for the prediction of SCD, cardiac mortality, or severe arrhythmic events at follow-up. After demonstration with Cochran's Q test and $\mathrm{I}^{2}$ statistic of the homogeneity of the results, we used a random effects model developed by DerSimonian and Laird [24]. Publication bias was evaluated with the use of Begg's test. Finally, we performed sensitivity analyses to examine the influence of each study on the pooled estimate by omitting each study one at a time. All statistical tests were 2-sided and were evaluated at a significance level of 0.05. STATA version 12.0 (College Station, TX, USA) was used to conduct all analyses.

\section{Results}

\section{Study characteristics}

An initial literature search using the previously mentioned search terms identified 963 potential articles for inclusion in this study. After reviewing and eliminating clearly ineligible studies, we identified 5 prospective studies involving a total of 1,588 participants (Figure 1) [18-22]. One other study did not provide enough primary data [23], an attempt was made to contact the author, but failed to receive the original data. Therefore, this study was not included in our current analysis. All studies were identified in MEDLINE, although some were also found in other databases. All studies were classified as being of good quality.

\section{Predictive accuracy of AECG-based TWA}

The baseline characteristics of included studies, stratified by the study of origin, are presented in Table 1 . The studies enrolled an overall number of 1,588 patients who were followed up for an average of 33 months. Overall, mean age was 64 years, $74 \%$ of patients were men, and approximately $72 \%$ of patients had underlying ischemic heart disease. A positive TWA result observed overall in $317(20 \%)$ of the patients (Table 2), 87 (28\%) patients reached the endpoint. Whereas a negative result observed in $1,271(80 \%)$ of the patients (Table 2), 120 (9\%) patients reached the endpoint.

In the study by Monasterio et al. (Table 3) [22], for the end point of cardiac mortality, the prognostic value of TWA was negative (HR: 1.44, 95\% CI: 0.97-2.13). For

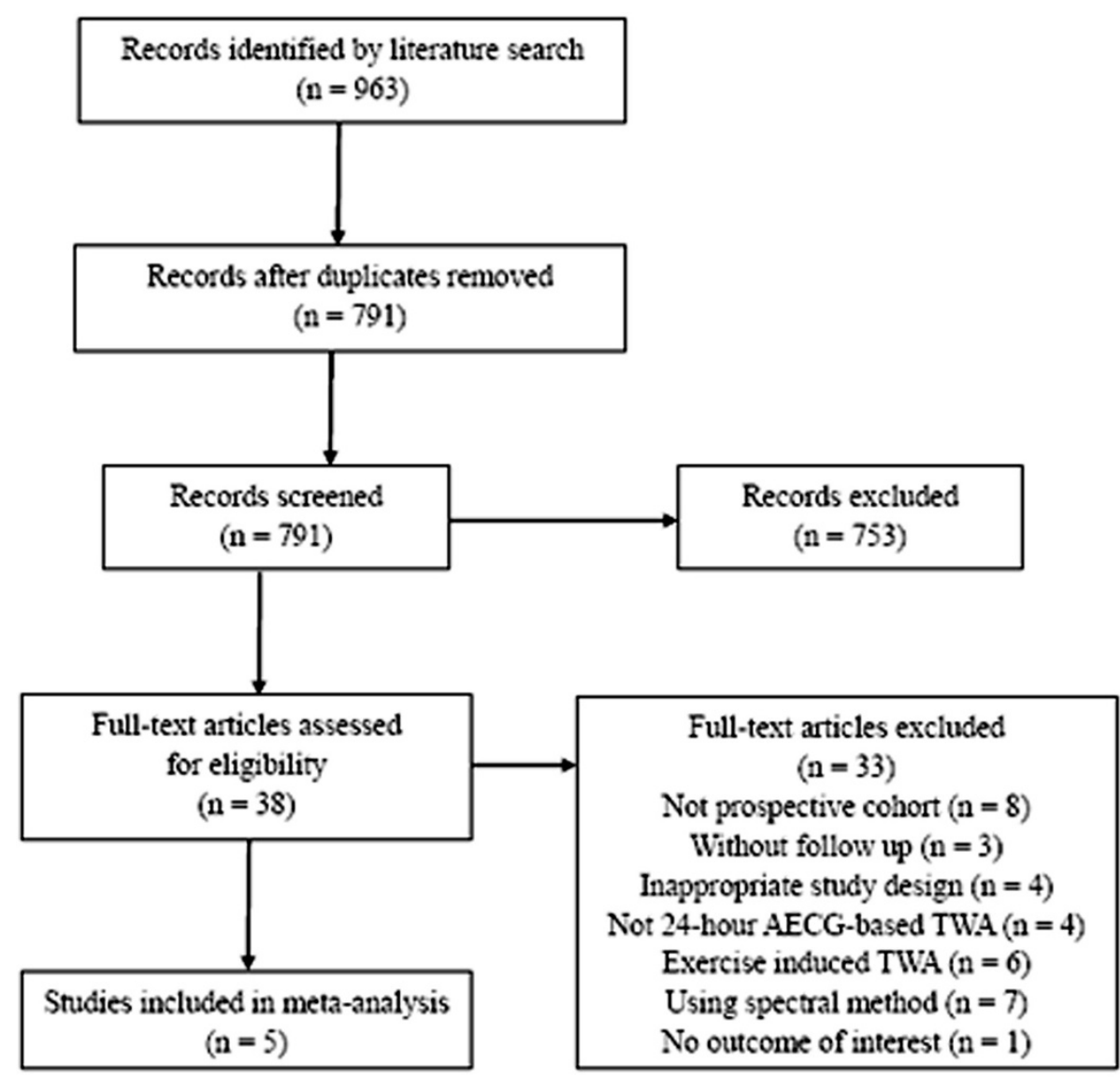

Figure 1 PRISMA flow diagram. Summary of the evidence search and selection. 
Table 1 Characteristics of prospective cohort studies of AECG-based TWA

\begin{tabular}{|c|c|c|c|c|c|c|c|}
\hline Study & Total (n) & Mean age (yrs) & Men (\%) & ICD implanted (\%) & Population & Mean LVEF (\%) & $\begin{array}{l}\text { Average follow-up } \\
\text { (months) }\end{array}$ \\
\hline Sakaki et al. 2009 [19] & 295 & 66 & 72 & 7 & $\begin{array}{c}\text { LVEF }<40 \% \text {, dilated } \\
\text { left ventricle }\end{array}$ & 34 & 13 \\
\hline Sulimov et al. 2012 [20] & 111 & 64 & 76 & - & Post-Ml & 46.6 & 12 \\
\hline Yu et al. 2012 [21] & 219 & 56 & 81 & 0 & Acute Ml & $>35 \%{ }^{*}$ & 16 \\
\hline $\begin{array}{l}\text { Monasterio et al. } \\
2012 \text { [22] }\end{array}$ & 650 & 63 & 71 & - & $\begin{array}{l}\text { symptomatic CHF } \\
\text { corresponding to } \\
\text { NYHA classes II and III }\end{array}$ & $<35 \%^{\dagger}$ & 48 \\
\hline Hoshida et al. 2013 [18] & 313 & 70 & 74 & 1 & Post-Ml & 47 & 39 \\
\hline
\end{tabular}

Abbreviations: AECG ambulatory electrocardiogram, CHF chronic heart failure, LVEF left ventricular ejection fraction, MI myocardial infarction, NYHA New York Heart Association, - not stated. ${ }^{*}$ Ninety-two percent patients had LVEF greater than $35 \%$. ${ }^{\dagger}$ Fifty-five percent patients had LVEF smaller than $35 \%$.

the end point of SCD, the prognostic value of TWA was positive (HR: 2.29, 95\% CI: $1.31-4.00$ ).

We pooled data across studies to investigate the predictive ability of TWA (Tables 2 and 3). In our primary analysis of the composite endpoint of SCD, cardiac mortality, and severe arrhythmic events, the risk of patients with positive TWA results was significantly increased compared to negative results (HR: 5.94, 95\% CI: 1.80 to 19.63) (Figure 2, Table 3). Subgroup summary estimates showed that TWA-positive outcome was associated with increased risk of SCD (HR: 7.49, 95\% CI: 2.65 to 21.15) and cardiac mortality (HR: $4.75,95 \% \mathrm{CI}: 0.42$ to 53.55 ) (Table 3). Four studies evaluating TWA measured using the modified moving average (MMA) method, MMATWA-positive outcome was significantly associated with the composite endpoint (SCD, cardiac mortality, and severe arrhythmic events, HR: 9.51, 95\% CI: 4.99 to 18.11).

\section{Discussion}

We conducted the first meta-analysis of 24-hour AECGbased TWA as a predictor of cardiac events in a wide variety of patient populations, accumulating prospective data on more than 1,500 subjects. Overall, we have found that a positive TWA result predicted nearly six-fold risk of composite endpoint (SCD, cardiac mortality, and severe arrhythmic events) compared with a negative result.

As for the study that didn't provide HR [23], 199 patients (mean age was 62 years, mean LVEF was $45 \%$ ) with AMI undergoing 24-hour Holter ECG-based TWA assessment using the modified moving average (MMA) method. The primary outcome was sudden death and sustained ventricular arrhythmia treated with external electric cardioversion during 6-month follow-up period. TWA was positive in $8.5 \%$ patients (cut-off value was 46 $\mu \mathrm{V})$. Survival analyses using Cox survival models revealed that TWA was not significantly associated with the incidence of the primary outcome $(\mathrm{P}=0.15)$.

Studies with exercise-based and AECG-based TWA have shown that an abnormal TWA test indicates an increased risk for arrhythmic events or mortality [1-3,5]. Studies with the exercise-based TWA is more extensive $[1,2]$. However, measurement of exercise-based TWA using spectral method requires a specialized protocol and a target heart rate range of 105 to 110 beats/min for $>2 \min [1,6]$. As a result, spectral method has limited applicability in patients with heart failure or undertake beta-blocking agents. For studies with high ineligible rate (49\% to $53 \%)$ of spectral method-based TWA test results in decompensated heart failure [7] and post-AMI [8] patients, the incomplete TWA test was the most significant predictor of mortality. Moreover, of the patients able to perform the test, TWA did not predict mortality in both studies $[7,8]$. Alternative strategies would be required for the significant proportion of ineligible patients.

Furthermore, spectral method has a relatively high incidence (19\% to $46 \%)$ of indeterminate test results [1,7,9-11], rising debating on the interpretation of TWA test results. The interpretation of indeterminate TWA in

Table 2 Test characteristics of AECG-based TWA

\begin{tabular}{|c|c|c|c|c|c|c|c|c|}
\hline Study & End Point & HR (95\% Cl) & $P$ value & TWA Pos \% & TWA Neg \% & Cut-off value $(\mu \mathrm{V})$ & Lead & Method \\
\hline Sakaki et al. 2009 [19] & Cardiac mortality $^{*}$ & $17.1(6.3-46.6)$ & $P<0.0001$ & 18 & 82 & 65 & V1 or V5 & MMA \\
\hline Sulimov et al. 2012 [20] & SCD & $5.01(1.5-17.0)$ & 0.005 & 41 & 59 & 53.5 & - & MMA \\
\hline Yu et al. 2012 [21] & SCD & $15.07(2.88-78.68)$ & 0.0031 & 22 & 88 & 47 & V2 or V5 & MMA \\
\hline $\begin{array}{l}\text { Monasterio et al. } \\
2012 \text { [22] }\end{array}$ & Cardiac mortality & $1.44(0.97-2.13)$ & 0.068 & 24 & 76 & 3 & - & LLR \\
\hline Hoshida et al. 2013 [18] & Fatal arrhythmic events & $5.8(1.6-20.8)$ & 0.0072 & 5 & 95 & 64 & V1 or V5 & MMA \\
\hline
\end{tabular}

Abbreviations: AECG ambulatory electrocardiogram, HR hazard ratio, MMA modified moving average, Neg negative, Pos positive, SCD sudden cardiac death, - not stated. ${ }^{*}$ One patient who received ICD defibrillation therapy was included. 
Table 3 Combined HR of AECG-based TWA related to fatal cardiac events by endpoint and study method

\begin{tabular}{lcc}
\hline Group & No. of studies & HR (95\% Cl) \\
\hline $\begin{array}{l}\text { Composite endpoint: SCD, } \\
\text { cardiac mortality, and severe } \\
\text { arrhythmic events }\end{array}$ & 5 & 5.94 (1.80 to 19.63) \\
Endpoint: SCD & & \\
$\quad$ Sulimov et al. & & \\
$\quad$ Yu et al. & & \\
Endpoint: Cardiac mortality & 2.65 to 21.15) \\
$\quad$ Sakaki et al. & 2 & \\
$\quad$ Monasterio et al. & & \\
Studies using MMA method & & \\
$\quad$ Sakaki et al. \\
$\quad$ Sulimov et al. \\
$\quad$ Yu et al. \\
$\quad$ Hoshida et al.
\end{tabular}

Abbreviations: AECG ambulatory electrocardiogram, $H R$ hazard ratio, $M M A$ modified moving average, $S C D$ sudden cardiac death.

the individual patient must be integrated with the clinical history of that patient $[1,9]$. Recordings with respiration, muscle, or other motion artifacts or electrode noise provide no prognostic information [11]. Inconsistency remains in current practice as to whether an indeterminate TWA test should be considered abnormal [7-10,12-14].

Unlike the spectral method, AECG recording-based TWA could be analyzed in 24-hour Holter recording with daily activity, risk stratification is based on the peak TWA value, which permits visual examination to verify the presence and magnitude of TWA [15,25]. AECG recording-based TWA does not require elevation of heart rate to a target level, it affords an opportunity to assess cardiac electrical instability in patients who cannot exercise or taking beta-blocking agents $[5,16,17]$. Indeterminate results are infrequent ( $3 \%$ to $5 \%$ ) as no target heart rate is needed [2]. It remains important to use AECGbased TWA to risk stratify the patients who are ineligible for exercise-induced TWA test or have an indeterminate result. Furthermore, AECG analysis allows patients to be monitored for TWA during daily activity, incorporates the influences of heightened sympathetic nerve activity, disturbed nighttime breathing, and episodes of intense physiological or mental stress, which cannot be replicated during exercise stress tests.

Of the 5 included studies, 4 studies used the MMA method [18-21], one study used the Laplacian likelihood ratio (LLR) method [22]. Recently, Orini et al. performed a simulation study in vivo in humans to compare the accuracy of four commonly used methodologies, including spectral, MMA and LLR methods, for T-wave alternans mapping in electrograms [26]. They found that all methodologies provided accurate electrogram-TWA estimation/detection in ideal conditions, while LLR was the most accurate and robust, providing better detectionrates in noisy conditions.

MMA is the most used method employed for measures of AECG-based TWA in clinical studies $[1,15]$. There are two standard cut-points for MMA: $47 \mu \mathrm{V}$, which indicates "abnormal" and $60 \mu \mathrm{V}$ which indicates "severely abnormal" test results [1]. Recently, it has become possible to measure AECG-based TWA amplitude precisely [5]. It provides an opportunity for physicians to use TWA magnitude in risk assessment.

Previously, a meta-analysis performed by Rizas et al. shows that TWA using MMA method is powerful predictor of mortality in post-MI patients (HR: 5.53,

\begin{tabular}{|c|c|c|c|}
\hline Study & & HR $(95 \% \mathrm{Cl})$ & Weight $\%$ \\
\hline Sakaki et al & & $17.10(6.30 .46 .60)$ & 20.88 \\
\hline Sulimov et al & & $5.01(1.50 .17 .00)$ & 19.53 \\
\hline Yu et al & & $15.07(2.88 .78 .68)$ & 16.68 \\
\hline Monasterio et al & $\rightarrow$ & $1.44(0.97 .2 .13)$ & 23.83 \\
\hline Hoshida et al & & $5.80(1.60 .20 .80)$ & 19.09 \\
\hline Overall (I-squared $=86.3 \%, p=0.000$ ) & & $5.94(1.80 .19 .63)$ & 100.00 \\
\hline $\begin{array}{c}1 \\
.0127\end{array}$ & 1 & $\begin{array}{l}1 \\
8.7\end{array}$ & \\
\hline
\end{tabular}


95\% CI: 3.14 to 9.72) [27]. For the four clinical studies included in their study, there are both case-control and retrospective studies. Furthermore, TWA has been measured based on exercise or 24-hour AECG. There are several strengths of our meta-analysis of TWA. First, all of the studies analyzed the TWA based on the 24-hour AECG. Second, we assessed the predictive value of AECGbased TWA in a wide variety of patient populations, including both ischemic and non-ischemic cardiomyopathy with a wide range of LVEF. Finally, in contrast to many earlier TWA studies, we used SCD, cardiac mortality, and severe arrhythmic events, rather than all-cause mortality, as the endpoint.

\section{Study limitations}

There are several limitations to this study. First, all data were derived from prospective cohort studies of AECGbased TWA. We did not identify any randomized studies meeting our inclusion criteria. Second, the 5 included studies varied in study design, patient characteristics and duration of follow-up. Nevertheless, there was no statistical evidence of heterogeneity. Third, the end points of the individual studies used in the summary calculations were slightly different (e.g., cardiac mortality vs. SCD vs. fatal arrhythmic events) and may have introduced some inconsistency when comparing across studies. Finally, there were not sufficient data contained in the included studies to determine the incremental prognostic value of AECG-based TWA independent of other predictors of arrhythmic events, such as heart rate variability, heart rate turbulence, baroreflex sensitivity, or LVEF. Although AECG-based TWA seems clinically useful in this metaanalysis, the additional prognostic value of AECG-based TWA when used with other predictors of risk stratification is unclear.

\section{Conclusions}

We studied the use of AECG-based TWA as a predictor of cardiac events in a meta-analysis of more than 1,500 subjects. We found that the positive TWA result predicted a nearly six-fold risk of a cardiac event compared with the negative result. Given the challenge of risk stratification for fatal cardiac events that faces today's cardiologist and the economic implications for today's healthcare system, our study helps understand the value and the limitations of AECG-based TWA.

\section{Additional file}

\section{Additional file 1: PRISMA 2009 Checklist.}

\section{Competing interests}

The authors declare that they have no competing interests.

\section{Authors' contributions}

Conception/Design: X-QQ, H-LZ, C-TZ. Collection and/or assembly of data: X-QQ, $H-L Z$, LR. Data analysis and interpretation: LR, J-GL, J-HY, FY, KH. Manuscript writing: $\mathrm{X}-\mathrm{QQ}, \mathrm{H}-\mathrm{LZ}$. All authors reviewed the manuscript and contributed to revising and approving the content of the final version for submission for publication.

\section{Acknowledgements}

This work is supported by the grants from the National Natural Science Foundation of China (81400255), the Independent Innovation Program of the Huazhong University of Science and Technology (01-18-540195) and the Scientific and Technological Projects of Hubei (2013CFB081, 2014CFB412). The funders had no role in the study design, data collection, analysis, decision to publish, or preparation of the manuscript. The corresponding author had full access to all the data in the study and had final responsibility for the decision to submit for publication.

\section{Author details}

${ }^{1}$ Department of Geriatrics, Tongji Hospital, Tongji Medical College, Huazhong University of Science and Technology, Wuhan 430030, China. ${ }^{2}$ Department of Cardiology, Tongji Hospital, Tongji Medical College, Huazhong University of Science and Technology, Wuhan 430030, China. ${ }^{3}$ Department of Cardiology, Tongji ShenZhi Hospital, Wuhan, China.

Received: 19 October 2014 Accepted: 9 December 2014

Published: 20 December 2014

\section{References}

1. Verrier RL, Klingenheben T, Malik M, El-Sherif N, Exner DV, Hohnloser SH, Ikeda T, Martinez JP, Narayan SM, Nieminen T, Rosenbaum DS: Microvolt T-wave alternans physiological basis, methods of measurement, and clinical utilityconsensus guideline by International Society for Holter and Noninvasive Electrocardiology. J Am Coll Cardiol 2011, 58(13):1309-1324.

2. Verrier RL, Malik M: Clinical applications of T-wave alternans assessed during exercise stress testing and ambulatory ECG monitoring. J Electrocardio/ 2013, 46(6):585-590.

3. van der Avoort CJ, Filion KB, Dendukuri N, Brophy JM: Microvolt T-wave alternans as a predictor of mortality and severe arrhythmias in patients with left-ventricular dysfunction: a systematic review and meta-analysis. BMC Cardiovasc Disord 2009, 9:5.

4. Cieplucha A, Trojnarska O, Bartczak A, Kramer L, Grajek S: Microvolt T wave alternans in adults with congenital heart diseases characterized by right ventricle pathology or single ventricle physiology: a case control study. BMC Cardiovasc Disord 2013, 13:26.

5. Verrier RL, Ikeda T: Ambulatory ECG-based T-wave alternans monitoring for risk assessment and guiding medical therapy: mechanisms and clinical applications. Prog Cardiovasc Dis 2013, 56(2):172-185.

6. Rosenbaum DS, Jackson LE, Smith JM, Garan H, Ruskin JN, Cohen RJ: Electrical alternans and vulnerability to ventricular arrhythmias. N Engl J Med 1994, 330(4):235-241.

7. Jackson CE, Myles RC, Tsorlalis IK, Dalzell JR, Rocchiccioli JP, Rodgers JR, Spooner RJ, Greenlaw N, Ford I, Gardner RS, Cobbe SM, Petrie MC, McMurray $\mathrm{J}$ : Spectral microvolt T-wave alternans testing has no prognostic value in patients recently hospitalized with decompensated heart failure. Eur J Heart Fail 2013, 15(11):1253-1261.

8. Tapanainen JM, Still AM, Airaksinen KE, Huikuri HV: Prognostic significance of risk stratifiers of mortality, including $T$ wave alternans, after acute myocardial infarction: results of a prospective follow-up study. J Cardiovasc Electrophysiol 2001, 12(6):645-652.

9. Gehi AK, Stein RH, Metz LD, Gomes JA: Microvolt T-wave alternans for the risk stratification of ventricular tachyarrhythmic events: a meta-analysis. J Am Coll Cardiol 2005, 46(1):75-82.

10. Kaufman ES, Bloomfield DM, Steinman RC, Namerow PB, Costantini O, Cohen RJ, Bigger JT Jr: "Indeterminate" microvolt T-wave alternans tests predict high risk of death or sustained ventricular arrhythmias in patients with left ventricular dysfunction. J Am Coll Cardiol 2006, 48(7):1399-1404.

11. Chan PS, Bartone C, Booth T, Kereiakes D, Chow T: Prognostic implication of redefining indeterminate microvolt T-wave alternans studies as abnormal or normal. Am Heart J 2007, 153(4):523-529.

12. Bloomfield DM, Steinman RC, Namerow PB, Parides M, Davidenko J, Kaufman ES, Shinn T, Curtis A, Fontaine J, Holmes D, Russo A, Tang C, Bigger JT Jr: Microvolt T-wave alternans distinguishes between patients 
likely and patients not likely to benefit from implanted cardiac defibrillator therapy: a solution to the Multicenter Automatic Defibrillator Implantation Trial (MADIT) II conundrum. Circulation 2004, 110(14):1885-1889.

13. Gold MR, Ip JH, Costantini O, Poole JE, McNulty S, Mark DB, Lee KL, Bardy GH: Role of microvolt T-wave alternans in assessment of arrhythmia vulnerability among patients with heart failure and systolic dysfunction: primary results from the T-wave alternans sudden cardiac death in heart failure trial substudy. Circulation 2008, 118(20):2022-2028,

14. Costantini O, Hohnloser SH, Kirk MM, Lerman BB, Baker JH 2nd, Sethuraman B, Dettmer MM, Rosenbaum DS, Investigators AT: The ABCD (Alternans Before Cardioverter Defibrillator) Trial: strategies using T-wave alternans to improve efficiency of sudden cardiac death prevention. J Am Coll Cardiol 2009, 53(6):471-479.

15. Nearing BD, Verrier RL: Modified moving average analysis of T-wave alternans to predict ventricular fibrillation with high accuracy. J Appl Physiol (1985) 2002, 92(2):541-549.

16. Verrier RL, Kumar K, Nearing BD: Basis for sudden cardiac death prediction by T-wave alternans from an integrative physiology perspective. Heart Rhythm 2009, 6(3):416-422.

17. Li-na R, Xin-hui F, Li-dong R, Jian G, Yong-quan W, Guo-xian Q: Ambulatory ECG-based T-wave alternans and heart rate turbulence can predict cardiac mortality in patients with myocardial infarction with or without diabetes mellitus. Cardiovasc Diabetol 2012, 11:104.

18. Hoshida K, Miwa Y, Miyakoshi M, Tsukada T, Yusu S, Yoshino H, Ikeda T: Simultaneous assessment of T-wave alternans and heart rate turbulence on holter electrocardiograms as predictors for serious cardiac events in patients after myocardial infarction. Circ J 2013, 77(2):432-438.

19. Sakaki K, Ikeda T, Miwa Y, Miyakoshi M, Abe A, Tsukada T, Ishiguro H, Mera H, Yusu S, Yoshino H: Time-domain T-wave alternans measured from Holter electrocardiograms predicts cardiac mortality in patients with left ventricular dysfunction: a prospective study. Heart Rhythm 2009, 6(3):332-337.

20. Sulimov V, Okisheva E, Tsaregorodtsev D: Non-invasive risk stratification for sudden cardiac death by heart rate turbulence and microvolt T-wave alternans in patients after myocardial infarction. Europace 2012, 14(12):1786-1792.

21. Yu H, Pi-Hua F, Yuan W, Xiao-Feng L, Jun L, Zhi L, Sen L, Zhang S: Prediction of sudden cardiac death in patients after acute myocardial infarction using T-wave alternans: a prospective study. J Electrocardio/ 2012, 45(1):60-65.

22. Monasterio V, Laguna P, Cygankiewicz I, Vazquez R, Bayes-Genis A, de Luna AB, Martinez JP: Average T-wave alternans activity in ambulatory ECG records predicts sudden cardiac death in patients with chronic heart failure. Heart Rhythm 2012, 9(3):383-389.

23. Arisha MM, Girerd N, Chauveau S, Bresson D, Scridon A, Bonnefoy E, Chevalier P: In-hospital heart rate turbulence and microvolt T-wave alternans abnormalities for prediction of early life-threatening ventricular arrhythmia after acute myocardial infarction. Ann Noninvasive Electrocardiol 2013, 18(6):530-537.

24. DerSimonian R, Laird N: Meta-analysis in clinical trials. Control Clin Trials 1986, 7(3):177-188.

25. Stein PK, Sanghavi D, Domitrovich PP, Mackey RA, Deedwania P: Ambulatory ECG-based T-wave alternans predicts sudden cardiac death in high-risk post-MI patients with left ventricular dysfunction in the EPHESUS study. J Cardiovasc Electrophysiol 2008, 19(10):1037-1042.

26. Orini M, Hanson B, Monasterio V, Martinez JP, Hayward M, Taggart P, Lambiase P: Comparative evaluation of methodologies for T-wave alternans mapping in electrograms. IEEE Trans Biomed Eng 2014, 61(2):308-316.

27. Rizas KD, Bauer A: Risk stratification after myocardial infarction: it is time for intervention. Europace 2012, 14(12):1684-1686.

doi:10.1186/1471-2261-14-198

Cite this article as: Quan et al: Ability of ambulatory ECG-based T-wave alternans to modify risk assessment of cardiac events: a systematic review. BMC Cardiovascular Disorders 2014 14:198.

\section{Submit your next manuscript to BioMed Central and take full advantage of:}

- Convenient online submission

- Thorough peer review

- No space constraints or color figure charges

- Immediate publication on acceptance

- Inclusion in PubMed, CAS, Scopus and Google Scholar

- Research which is freely available for redistribution

Submit your manuscript at www.biomedcentral.com/submit
Ciomed Central 